\title{
Editorial
}

\section{Machine Learning in Transportation}

\author{
Ali Tizghadam $\mathbb{D}^{\mathbb{D}},{ }^{1}$ Hamzeh Khazaei, ${ }^{2}$ Mohammad H. Y. Moghaddam, ${ }^{3}$ and Yasser Hassan ${ }^{4}$ \\ ${ }^{1}$ TELUS \& University of Toronto, Toronto, Canada \\ ${ }^{2}$ University of Alberta, Edmonton, Canada \\ ${ }^{3}$ Ferdowsi University of Mashhad, Mashhad, Iran \\ ${ }^{4}$ Carlton University, Ottawa, Canada
}

Correspondence should be addressed to Ali Tizghadam; ali.tizghadam@telus.com

Received 21 May 2019; Accepted 21 May 2019; Published 4 June 2019

Copyright (C) 2019 Ali Tizghadam et al. This is an open access article distributed under the Creative Commons Attribution License, which permits unrestricted use, distribution, and reproduction in any medium, provided the original work is properly cited.

Nation's economy, safety, and quality of life are influenced by a well-behaved transportation system. Yet, demands in transportation are ever increasing due to trends in population growth, emerging technologies, and the increased globalization of the economy which has kept pushing the system to its limits. The rate of increasing the number of vehicles is at points even more than the overall population increase rate, which leads to more congested and dangerous roadways. This problem is not going to be addressed by just adding to the number of roads anymore. The construction cost is very high and the time to return the result is too lengthy to catch up with the vehicle increase rate.

One way to improve upon the fleet management is by viewing the road as an information highway as opposed to highway for vehicles. The scale of ingested data in the transportation system and even the interaction of various components of the system that generates the data have become a bottleneck for the traditional data analytics solutions. On the other hand, machine learning is a form of Artificial Intelligence (AI) and a data-driven solution that can cope with the new system requirements. Machine learning learns the latent patterns of historical data to model the behavior of a system and to respond accordingly in order to automate the analytical model building.

The availability of increased computational power and collection of the massive amount of data have redefined the value of the machine learning-based approaches for addressing the emerging demands and needs in transportation systems.
Machine learning solutions have already begun their promising marks in the transportation industry, where it is proved to even have a higher return on investment compared to the conventional solutions. However, the transportation problems are still rich in applying and leveraging machine learning techniques and need more consideration. The underlying goals for these solutions are to reduce congestion, improve safety and diminish human errors, mitigate unfavorable environmental impacts, optimize energy performance, and improve the productivity and efficiency of surface transportation.

In this special issue, we present original research work aimed at reporting on new models, algorithms, and case studies related to the use of machine learning in the field of transportation and further analysis of the reliability and robustness of the whole transportation system. In particular, the special issue focuses on prediction methods in transportation, transport network traffic flows and signals, public transportation including air fleet, driving styles, electric cars, and car sharing.

In recent years, machine learning techniques have become an integral part of realizing smart transportation. In this context, using an improved deep learning model, the complex interactions among roadways, transportation traffic, environmental elements, and traffic crashes have been explored. The proposed model includes two modules, an unsupervised feature learning module to identify functional relationship between the explanatory variables and the feature representations and a supervised fine-tuning module to 
perform traffic crash prediction. Another study leverages a hierarchical traffic signal control algorithm based on reinforcement learning, to solve the problem of "green wave bottleneck point" of the arterial and signal interference.

No effective travel guidance can be provided without a good travel time estimation strategy. Travel time can be achieved directly or indirectly. Direct methods measure travel time using probe vehicle, records at toll stations, tracking of cell phones, and many other technologies. Indirect methods infer travel time using measured traffic volume, speed, and occupancy in point sensors (e.g., loop detector, video camera) along the vehicle trajectory. In recent years, data mining and machine learning have gradually come into sight. The development of traffic information acquisition technologies (such as data of GPS trajectories) has provided us with a large amount of traffic data, which in turn paves the road to develop a more accurate travel time estimation based on data mining. Compared with traditional parametric models, data mining algorithms can explore implicit relationships between variables. To this end, in the special issue, a new data mining technique is introduced to leverage Random Forests for travel time estimation. It is shown that the influence of variables on travel time can be deeply excavated through Random Forests.

In Intelligent Transport Networks (ITS) context, accurate prediction of future traffic conditions is essential to mitigate traffic congestion and to respond to the traffic incidents. In the special issue, a hybrid traffic flow prediction method is proposed based on k-nearest neighbor (KNN) algorithm and long short-term memory network (LSTM). The improved accuracy in traffic flow prediction is illustrated through traffic flow prediction at two stations using detector data on major freeways/expressways in Minnesota.

Along the same lines, Unmanned Aerial Vehicles (UAVs) have great potential in daily traffic management and control as well as collection of traffic condition data at a low cost. However, UAV comes with its own challenges, primarily related to vehicle size in the images and potential occlusion by shadows or other road objects, especially in congested traffic conditions. In this context, two learning-based frameworks are proposed to detect vehicles and track vehicle trajectories in congested traffic conditions.

Statistical machine learning algorithms have also found their way in supporting smart transportation. In one of the research works reported in the special issue, using neighborhood components analysis and the Bayesian optimization algorithm, a random forest model has been trained to estimate the traffic incident duration with high accuracy. In another study in the context of statistical machine learning for transportation, a naive Bayesian classifier is used to identify public transit commuters' travel pattern based on both the smartcard and survey data sampled from commuters.

In a related work on public transit, a system architecture based on acceleration sensor data is proposed to determine the identity of the public transport driver. The proposed system is comprised of three major modules: data collection, data preprocessing and driver identification modules. The data is collected from active campus shuttle buses, the preprocessing module applies a filter to remove periods of inactivity from the data, and to extract the unique behavior of the driver (which results in identifying the driver), histogram of acceleration sensor data is proposed.

Demand for air travel continues to grow at a rapid pace; consequently, air traffic control is becoming more complex. The main challenge in air travel remains to be the collision avoidance, and given the increase in demand, automated decision support technologies, mainly Conflict Detection Resolution (CDR), will be required to enable continued provisioning of safe and efficient services in increasingly congested skies. In the special case of Free Flight, pilots have the freedom to choose their trajectory and speed to optimize the flight duration and performance while maintaining safe separation from neighboring traffic. To this end, it is important to find a fast conflict detection method in Free Flight, which is applicable in terminals as well as enroute. An attempt to address this problem is presented in which the aircraft conflict detection is considered as a binary classification problem and a pattern recognition approach is used to solve it. The flight data features are extracted and fed into a classifier which has been trained by a large flight dataset. A support vector machine (SVM) method is employed to detect multi-aircraft conflict in Free Flight airspace and to detect the conflict probability. False alarm rate is the most important issue for air traffic controllers, and the proposed method uses Synthetic Minority Oversampling Technique (SMOTE) to handle imbalanced datasets.

Classification techniques have found their way in a more general sense in transportation networks. Learning the latent patterns of historical data in an efficient way to model a transportation system is a major need for making right decisions. However, many classification algorithms in the literature assume that the largest values in the datasets are unordered, so they lose inherent order between the class values. To tackle this problem, a research study is proposed to leverage Ensemble-Based Ordinal Classification (EBOC) approach, which suggests bagging and boosting, AdaBoost algorithm, methods as a solution for ordinal classification problem in transportation. The proposed method is compared with more traditional tree-based classification algorithms such as Decision Tree and Random Tree.

Distracted driving has been widely reported to be a major contributing factor to traffic collisions. With the emergence of new learning-based methods, addressing the driver's distraction problem is becoming a topic of interest among industry and academics. In the special issue, we investigate methods to detect and mitigate the driving style using deep learning and utilizing RGB images obtained from a camera mounted above the dashboard. In the popular case of rear-end collision crash, a study is presented that is focused on developing a driving style recognition method based on vehicle trajectory data extracted from surveillance camera. The study uses reference KPIs such as Inversed Time to Collision (ITTC) and Time-Headway (THW) to evaluate the collision risk level of vehicle trajectory for each driver and we leverage Support Vector Machine (SVM) to recognize driving style. Moreover, the SVM approach is compared with different learning methods including Random Forest (RF), KNearest-Neighbors (KNN), and Multilayer Perceptron (MLP) in terms of the precision of driving style recognition. 
Car-sharing is becoming an increasingly popular travel mode around the globe. Placement problem is one of the most challenging and interesting issues to be addressed in the context of car sharing. Most of car-sharing station places are chosen either by experience or just randomly on places where the space has been available. This results in having low efficiency and consequently capital loss in many car-sharing stations. A study is included in the special issue aiming at using different data sources with statistical models and a machine learning algorithm to help car-sharing operator choose the optimal location of new stations and adjust the location of existing ones. In the study, the area of interest is assumed to be a grid, and the attention is given to find a model to estimate a potential travel demand value for each small grid and the demand volume is used to suggest best places for carsharing stations. The study has compared different learning methods and concluded that Logistic Regression with LASSO (Least Absolute Shrinking and Selection Operator) is the best method to estimate the probability of existence of demand in all grids. In addition to demands per grid, the study focuses on a more competitive market and finds the influential factors on order number. Suggestions on the optimal location of stations are given in consideration of competitors.

In summary, we believe that the published contents in this special issue contribute in moving the smart transportation field forward and open new avenues for further research on how machine learning can be leveraged to build more sustainable and safer smart cities.

\section{Conflicts of Interest}

The editors declare that there are no conflicts of interest in publishing this editorial.

Ali Tizghadam

Hamzeh Khazaei

Mohammad H. Y. Moghaddam

Yasser Hassan 


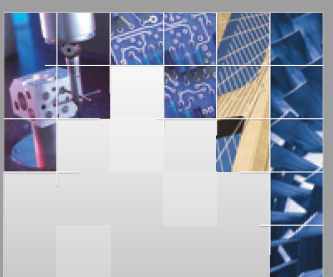

\section{Enfincering}
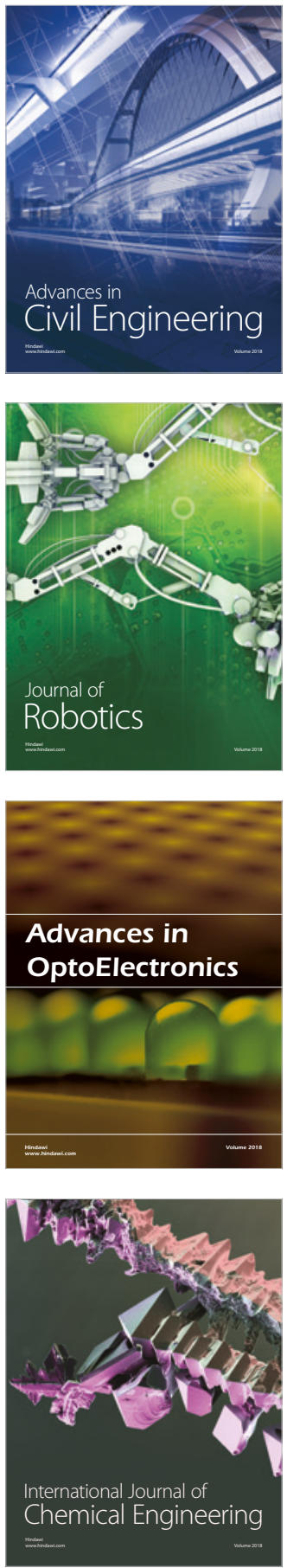

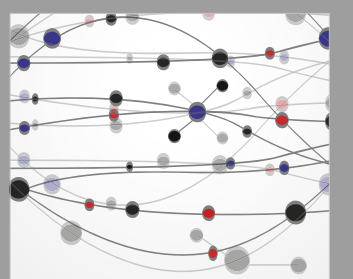

\section{Rotating \\ Machinery}

The Scientific World Journal

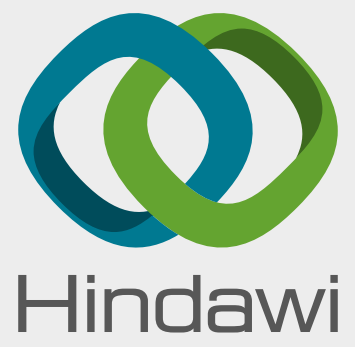

Submit your manuscripts at

www.hindawi.com
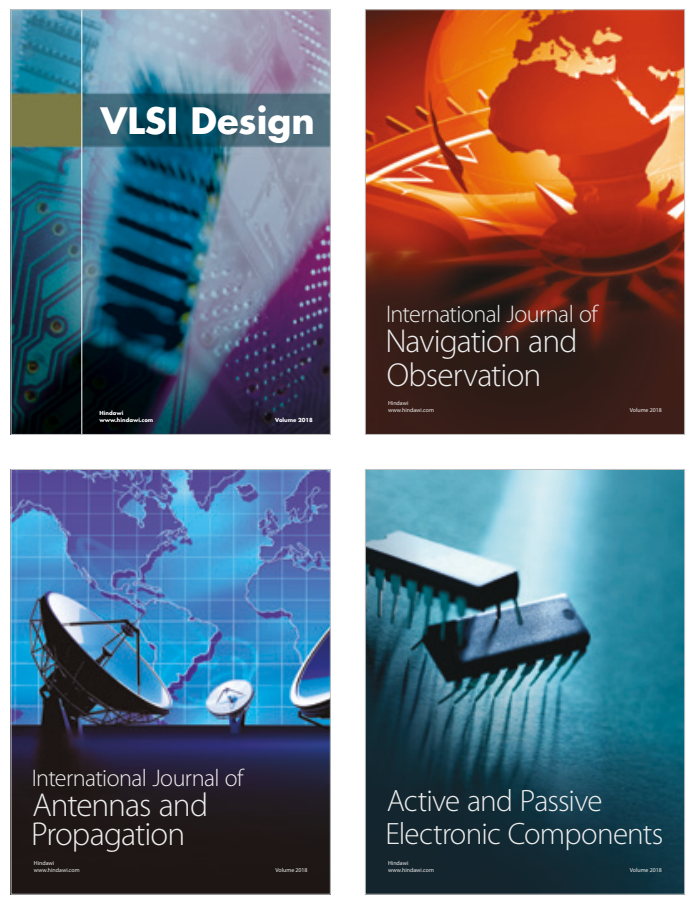
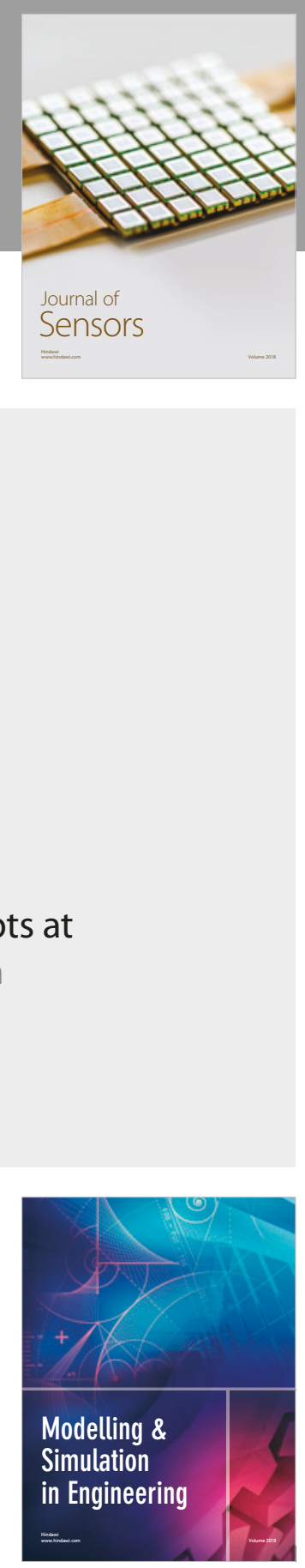

\section{Advances \\ Multimedia}
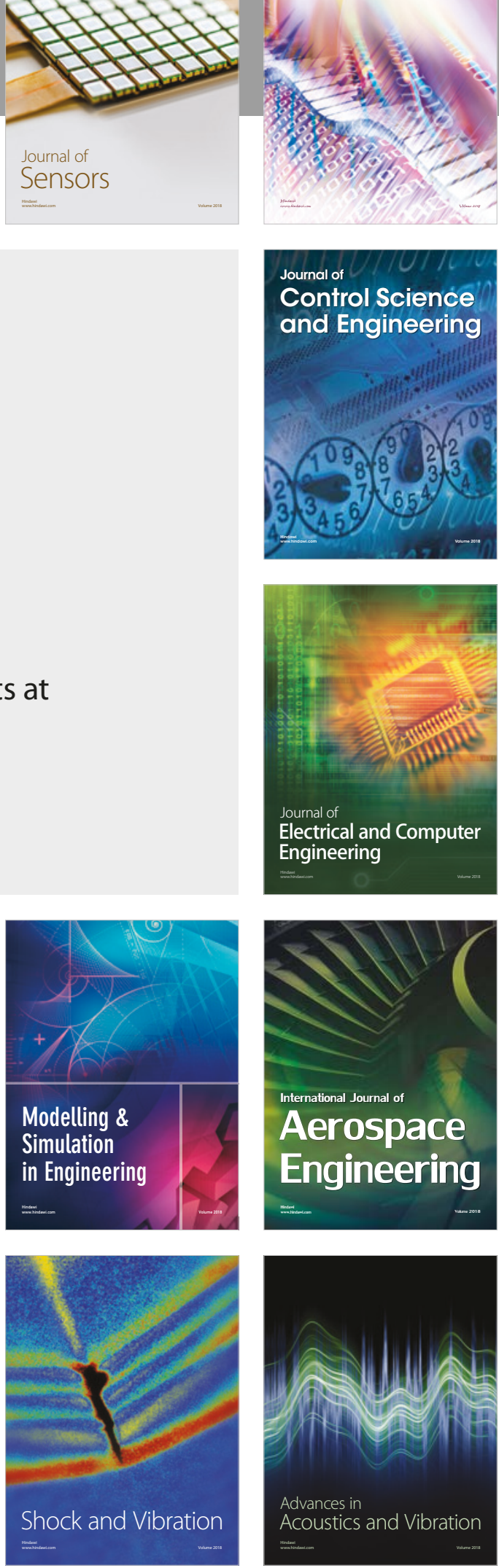